\title{
Neurological Sequelae Of Lupus
}

National Institute of Neurological Disorders and Stroke (NINDS)

\section{Source}

National Institute of Neurological Disorders and Stroke (NINDS). Neurological Sequelae

Of Lupus Information Page.

Lupus (also called systemic lupus erythematosus) is a disorder of the immune system.

Normally, the immune system protects the body against invading infections and cancers.

In lupus, the immune system is over-active and produces increased amounts of abnormal antibodies that attack the body's tissues and organs. Lupus can affect many parts of the body, including the joints, skin, kidneys, lungs, heart, nervous system, and blood vessels. The signs and symptoms of lupus differ from person to person; the disease can range from mild to life threatening.

Initial symptoms of lupus may beg in with a fever, vascular headaches, epilepsy, or psychoses. A striking feature of lupus is a butterfly shaped rash over the cheeks. In addition to headache, lupus can cause other neurological disorders, such as mild cognitive dysfunction, organic brain syndrome, peripheral neuropathies, sensory neuropathy, psychological problems (including personality changes, paranoia, mania, and schizophrenia), seizures, transverse myelitis, and paralysis and stroke. 